\title{
A Novel Spiking Cortical Model based Filter for Impulse Noise Removal
}

\author{
Xuming Zhang ${ }^{\mathrm{a}, *}$, Mingyue Ding ${ }^{\mathrm{a}}$, Yi Zhan ${ }^{\mathrm{a}}$, Yangchao Dou ${ }^{\mathrm{a}}$, Zhouping Yin ${ }^{\mathrm{b}}$ \\ ${ }^{a}$ School of Life Science and Technology, Huazhong University of Science and Technology, Wuhan, China \\ ${ }^{b}$ School of Mechanical Science and Engineering, Huazhong University of Science and Technology, Wuhan \\ China
}

\begin{abstract}
A novel spiking cortical model based switching mean filter for removing impulse noise is presented. In the proposed filter, the noise detector using spiking cortical model is first adopted to identify the pixels that are likely to be corrupted by impulse noise. Then the detected impulses are removed by the weighted mean filter while the noise-free pixels are left unaltered. Extensive simulations show that the proposed filter outperforms a number of existing decision-based filters due to its excellent performance in terms of effectiveness in image restoration. Because of its outstanding restoration performance, the proposed filter can be used for noise removal in numerous consumer electronics products such as digital camera and digital television.
\end{abstract}

Index Terms: impulse noise; spiking cortical model; noise detection; image restoration

(C) 2011 Published by MECS Publisher. Selection and/or peer review under responsibility of the Research Association of Modern Education and Computer Science.

\section{Introduction}

In the process of acquisition or transmission through such popular consumer electronics products as digital camera and digital television, digital images are likely to be contaminated by impulse noise. Impulse noise will result in image degradation by producing the significant intensity difference between the corrupted pixel and its local neighborhood. To improve the quality of degraded images in digital camera and digital television, it is of great significance to adopt effective approaches to remove impulse noise from digital images while preserving the details in the images.

The median (MED) filter has been widely used to remove impulse noise for its good noise suppression ability and high computational efficiency [1]. However, the traditional MED filter operates uniformly across the whole image regardless of whether the considered pixel is corrupted or not. To overcome the

* Corresponding author.

E-mail address: xmboshi.zhang@gmail.com 
disadvantages of the MED filter, the various switching based filters have been proposed, such as progressive switching median (PSM) filter [2], tri-state median (TSM) filter [3], non-recursive adaptive center weighted median (NACWM) filter [4], recursive adaptive center weighted median (RACWM) filter [4], Laplacian detector-based switching median (LDSM) filter[5], pixel-wise MAD-based (PWMAD) filter [6], fast switching median (FSM) filter [7] and opening-closing sequence (OCS) filter [8]. Such switching based filters involve two phases, namely, noise detection and noise removal. These filters generally perform better than the MED filter because of the introduction of noise detection, but they can not remove impulse noise effectively or can not preserve the image details very well when the images are highly corrupted.

The spiking cortical model (SCM), as a modified version of pulse-coupled neural network (PCNN), has been recently proposed and designed for image processing [9]. Derived from some other visual cortex models, the SCM has the characteristics of latency in information transfer and non-linear coupling modulation. The above attributes provide the SCM with great advantages over some traditional image processing methods, especially in the filed of image denoising, image enhancement, image segmentation and so on. In this paper, the SCM based filter is proposed to remove impulse noise from the digital images. The proposed filter can both realize accurate noise detection by means of SCM and suppress impulse noise while preserving the details in the images very well using the weighted mean filtering only for the detected impulses.

\section{SCM for Noise Detection}

It has been well known that the pixel corrupted by impulse noise has substantially higher or lower intensity value than its neighboring uncorrupted pixels. The great difference between corrupted pixels and noise-free pixels can be detected by the SCM because of its pulse coupling characteristics.

Let $I_{i, j}$ be the intensity value of the input noisy image at the pixel location $(i, j)$ and $S_{i, j}$ be stimulus (the normalized input image). In the SCM, the state oscillator, threshold oscillator and firing status of the ijth neuron are represented by $F_{i, j}, \Theta_{i, j}$ and $Y_{i, j}$, respectively. In the $n$th iteration of SCM, the above parameters can be computed as follows [9]:

$$
\begin{aligned}
& F_{i, j}[n]=f \cdot F_{i, j}[n-1]+S_{i j}+S_{i j} \cdot \sum_{k l} W_{i j k l} \cdot Y_{k l}[n-1] \\
& Y_{i, j}[n]=\left\{\begin{array}{lll}
1 & \text { if } \quad F_{i, j}[n]>\Theta_{i, j}[n-1] \\
0 & \text { otherwise }
\end{array}\right. \\
& \Theta_{i, j}[n]=g \cdot \Theta_{i, j}[n-1]+h \cdot Y_{i, j}[n]
\end{aligned}
$$

where $f$ and $g$ are decay constants which are less than 1 and $h$ are scalars. $W_{i j k l}$ is the connection function through which the neurons communicate.

From (1) (3), it can be seen clearly that the pixels with high intensity values will be fired before those with low intensity values. This characterisitics show that the impulses with high amplitude can be identified very well by choosing the suitable parameters involved in the SCM. It is easy to understand that the pixel $(i, j)$ will be regared as noise-free pixel with the noise flag $b_{i, j}=0$ if the corresponding neuron's firing status $Y_{i, j}[N]=0$ in the $N$ th iteration where $N$ denotes the maximum iteration times. But for the considered center pixel with $Y_{i, j}$ $[N]=1$, it can be identified as the noise pixel only if it is fired before the majority of pixels in the $\left(2 L_{d}+1\right) \times\left(2 L_{d}+1\right)$ detection window. Let $C_{i, j}[N]$ be the total number of the pixels with their corresponding neurons' firing status equal to 1 in the detection window. The ratio of the number of the pixels with their corresponding neurons fired to the total number of pixels in the detection window is defined as:

$$
R_{i, j}[N]=\frac{C_{i, j}[N]}{\left(2 L_{d}+1\right) \times\left(2 L_{d}+1\right)}
$$


The pixel $(i, j)$ with $Y_{i, j}[N]=1$ will be classified as the noise pixel with the noise flag $b_{i, j}=1$ if $R_{i, j}[N]<\lambda$, where $\lambda$ is a predefined threshold.

It should be noted that the above noise detection approach can not identify impulses with low amplitude. To address this problem, the input image will be transformed in the following way:

$$
I_{i, j}^{\prime}=I_{\max }-I_{i, j}
$$

where $I_{\max }$ denote the maximum pixel intensity value in the noisy images. The inversed image $I_{i, j}^{\prime}$ is normalized. The normalized result will be used as the stimulus of the SCM for identifying the impulses with low amplitude. Once the impulses with high amplitude and low amplitude have been identified, the noise detection results will be used for subsquent noise removal.

\section{Noise Removal}

The detected impulses will be removed by adaptive weighted mean filter. For the corrupted pixel $(i, j)$, the filtering window of size $\left(2 L_{f}+1\right) \times\left(2 L_{f}+1\right)$ is used. Starting with $L_{f}=1$, this filtering window iteratively extends outward by one pixel in its four sides until the number of noise-free pixels within this window is not less than 1 . Let $D_{i, j}$ denote the intensity values of noise-free pixels in the filtering window, i.e.,

$$
D_{i, j}=\left\{I_{i+s, j+t} \mid b_{i+s, j+t}=0, b_{i, j}=1,(s, t) \neq(0,0),-L_{f} \leq s, t \leq L_{f}\right\}
$$

The weighted mean value $g_{i, j}$ of the pixel values in $D_{i, j}$ is defined as:

$$
g_{i, j}=\frac{\sum_{f_{i s, j+t} \in D_{i, j}} w_{i+s, j+t} I_{i+s, j+t}}{\sum_{f_{i+s, j+t} \in D_{i, j}} w_{i+s, j+t}}
$$

where $w_{i+s, j+t}$ means the weight of $I_{i+s, j+t}$. Let $m_{i, j}$ be the median value of $D_{i, j}$. Because the median value has the least probability to be the value of the corrupted pixels, $m_{i, j}$ is utilized to determine $w_{i+s, j+t}$. It is easy to understand that the smaller the absolute difference between $I_{i+s, j+t}$ and $m_{i, j}$, the larger the weight $w_{i+s, j+t}$ should be to strengthen the influence of $I_{i+s, j+t}$ on $g_{i, j}$. Extensive simulations indicate that $w_{i+s, j+t}$ is greatly dependant on the above absolute difference and it should be decreasing with the increasing absolute difference. Accordingly, $w_{i+s, j+t}$ is chosen as:

$$
w_{i+s, j+t}=\frac{1}{1+\sqrt{\frac{\frac{\left|I_{i+s, j+t}-m_{i, j}\right|}{I_{\text {max }}-I_{\text {min }}}}{1-\frac{\left|I_{i+s, j+t}-m_{i, j}\right|}{I_{\max }-I_{\text {min }}}}}}
$$

where $I_{\min }$ denotes the minimum pixel intensity value in the noisy images.

The output of the SCM based filter is obtained by:

$$
h_{i, j}=b_{i, j} \cdot g_{i, j}+\left(1-b_{i, j}\right) I_{i, j}
$$

From (9), it can be seen that the noise-free pixel will not be altered while the detected impulse will be replaced by the weighted mean value of its neighboring noise-free pixels. 


\section{Simulations}

In this section, the proposed SCM based filter is compared with the MED filter, PSM filter, TSM filter, NACWM filter, RACWM filter, LDSM filter, PWMAD filter, OCS filter and FSM filter. The standard $512 \times$ 512 gray-level test image Mandrill is used for the experiments. Extensive simulations are conducted on the test image corrupted by the salt-pepper impulses with a wide range of noise densities varying from $10 \%$ to $80 \%$. In the SCM based filter, we set $f=0.9, g=0.8, F_{i, j}[0]=0, \Theta_{i, j}[0]=0.95, \lambda=0.6$ and $N=1$. Here the filtering performance is quantitatively measured by the peak signal-to-noise ratio (PSNR) and mean absolute difference (MAE) defined as:

$$
\begin{aligned}
\text { PSNR } & =10 \log _{10} \sum_{i=1}^{M} \sum_{j=1}^{N} \frac{255^{2}}{(o(i, j)-h(i, j))^{2}} d B \\
\text { MAE } & =\frac{\sum_{i=1}^{M} \sum_{j=1}^{N}|o(i, j)-h(i, j)|}{M \times N}
\end{aligned}
$$

where $o(i, j)$ denotes the intensity value of the original test image at the pixel location $(i, j)$ while $M$ and $N$ denote the total number of pixels in the horizontal and vertical dimensions of the image.

Table 1 and Table 2 list the PSNR values and MAE values of all the evaluated filters operating on the corrupted image Mandrill, respectively. It can be seen clearly from Tables 1 and 2 that the SCM based filter produces higher PSNR values and lower MAE values than all the other compared filters at the various noise ratios. Indeed, the objective evaluation based on PSNR and MAE measurement demonstrates that the SCM based filter has the best filtering performance among all these filters.

Tablel I. Filtering results in PSNR for the compared filters operating on the image mandrill

\begin{tabular}{|l|c|c|c|c|c|c|c|}
\hline \multirow{2}{*}{ Filters } & \multicolumn{7}{|c|}{ Noise density } \\
\cline { 2 - 8 } & $10 \%$ & $20 \%$ & $30 \%$ & $40 \%$ & $50 \%$ & $60 \%$ & $70 \%$ \\
\hline MED & 23.38 & 22.31 & 21.00 & 20.55 & 20.12 & 19.41 & 18.72 \\
\hline PSM & 27.95 & 25.38 & 23.98 & 22.77 & 21.92 & 21.14 & 20.03 \\
\hline TSM & 28.16 & 24.71 & 23.51 & 22.25 & 21.53 & 20.74 & 19.94 \\
\hline NACWM & 29.01 & 25.64 & 23.74 & 22.47 & 21.55 & 20.54 & 19.14 \\
\hline RACWM & 29.15 & 26.12 & 24.46 & 22.86 & 21.86 & 20.66 & 19.47 \\
\hline LDSM & 29.47 & 25.97 & 24.07 & 22.89 & 21.77 & 20.85 & 19.82 \\
\hline PWMAD & 28.42 & 24.85 & 23.17 & 22.21 & 21.28 & 20.45 & 19.46 \\
\hline OCS & 21.45 & 21.62 & 21.70 & 21.68 & 21.59 & 21.41 & 21.04 \\
\hline FSM & 27.30 & 26.12 & 25.00 & 23.61 & 22.55 & 21.47 & 20.69 \\
\hline SCM & 32.55 & 29.32 & 27.41 & 25.88 & 24.71 & 23.39 & 22.21 \\
\hline
\end{tabular}


Table II. Filtering results in PSNR for the compared filters operating on the image mandrill

\begin{tabular}{|c|r|r|r|r|r|r|c|}
\hline \multirow{2}{*}{ Filters } & \multicolumn{7}{|c|}{ Noise density } \\
\cline { 2 - 8 } & 10 & $20 \%$ & 30 & 40 & 50 & 60 & 70 \\
\hline MED & 10.623 & 11.709 & 15.064 & 15.752 & 16.967 & 17.941 & 19.436 \\
\hline PSM & 2.131 & 4.034 & 5.813 & 7.729 & 9.567 & 11.468 & 13.968 \\
\hline TSM & 2.110 & 4.679 & 6.423 & 8.696 & 10.472 & 12.856 & 15.064 \\
\hline NACW & 1.816 & 3.905 & 6.081 & 8.210 & 10.29 & 12.72 & 15.58 \\
\hline RACW & 1.793 & 3.778 & 5.544 & 7.716 & 9.908 & 12.43 & 15.74 \\
\hline LDSM & 1.793 & 3.778 & 5.544 & 7.716 & 9.908 & 12.436 & 15.748 \\
\hline PWMAD & 1.853 & 3.790 & 5.967 & 7.781 & 10.056 & 12.382 & 14.900 \\
\hline OCS & 1.935 & 4.423 & 6.635 & 8.735 & 10.673 & 12.955 & 15.816 \\
\hline FSM & 14.716 & 14.552 & 14.475 & 14.450 & 14.467 & 14.523 & 14.773 \\
\hline SCM & 1.278 & 2.631 & 3.985 & 5.466 & 7.024 & 8.917 & 10.899 \\
\hline
\end{tabular}

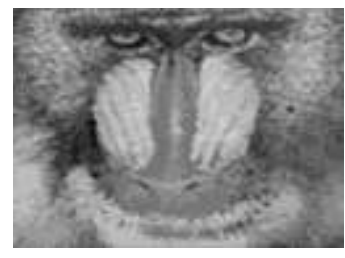

(g)

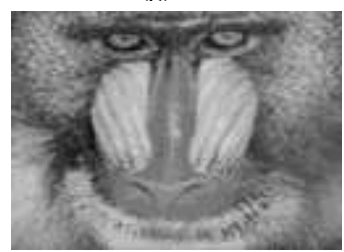

(i)

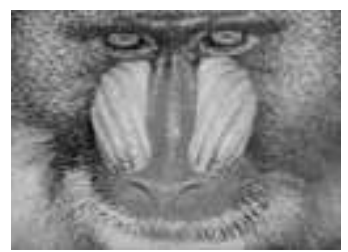

(k)

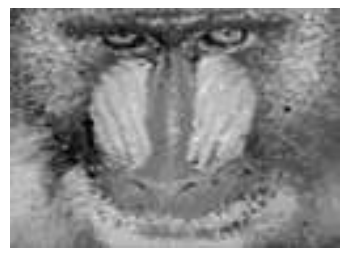

(h)

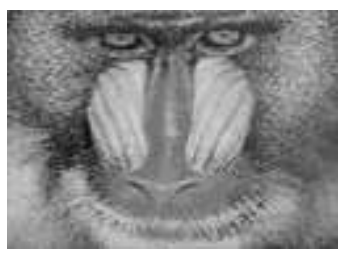

(i)

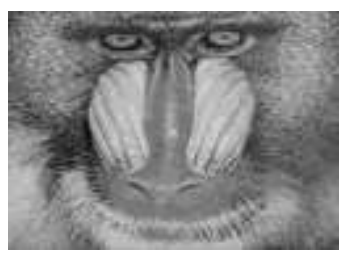

(1)

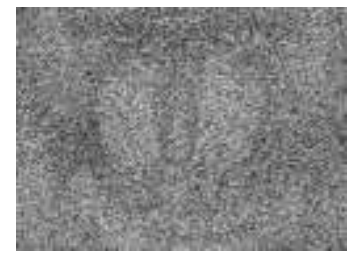

(a)

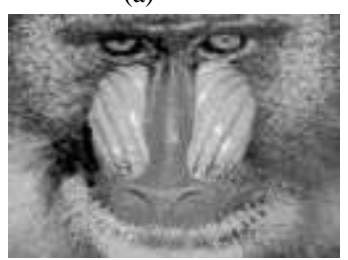

(c)

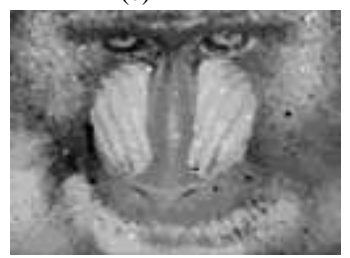

(e)

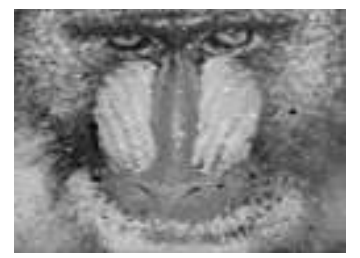

(b)

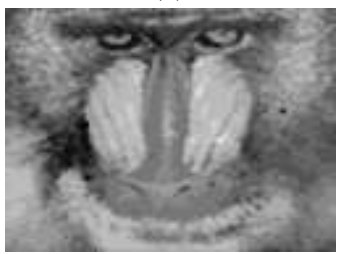

(d)

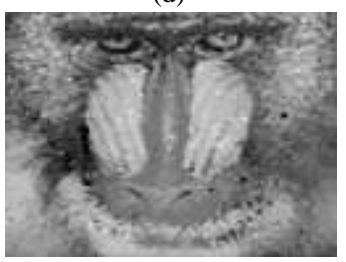

(f)

Fig. 1. Restoration results using the various filters for Mandrill corrupted by $70 \%$ salt-pepper impulses : (a) Corrupted Mandrill, (b) MED filter, (c) PSM filter, (d) TSM filter, (e) NCAWM filter, (f) RCAWM filter, (g) LDSM filter, (h) PWMAD filter, (i) OCS filter, (j) FSM filter,(k) SCM based filter, (l) Original Mandrill. 
The subjective visual comparisons are also made among the filtering results of all the compared filters. Fig. 1 shows the restoration results of these filters operating on the test image Mandrill corrupted by $70 \%$ saltpepper noise. Obviously, the MED filter, PSM filter, TSM filter, NACWM filter, RACWM filter, LDSM filter and PWMAD filters leave some impulses in the filtered images and lead to image blurring to some extent. The OCS filter and FSM filter cause the loss of some details although they suppress impulse noise better than the above filters. The SCM based filter provides better restoration results than other compared filters in that it not only removes impulse noise effectively but also preserves the fine details in the images very well.

\section{Conclusion}

In this paper, we proposed a simple SCM based impulse noise detector, which was combined with the weighted mean filter to remove impulse noise from the corrupted images. Experimentally, the proposed filter has been shown to significantly outperform numerous well-known decision-based filters by providing higher PSNR values and lower MAE values across a wide range of noise ratios. Indeed, the proposed filter will provide an efficient approach for suppressing impulse noise in digital cameras and digital television.

\section{Acknowledgements}

This research was supported by the National Natural Science Foundation of China under Grants No. 50625516 and was supported by the Fundamental Research Funds for the Central Universities, HUST: No. 2010JC036.

\section{References}

[1] R.C. Gonzalez, R.E. Woods, Digital image processing, 2nd ed, Englewood Cliffs, NJ: Prentice-Hall, 2001. [2] D. Zhang, "Progressive switching median filter for the removal of impulse noise from highly corrupted images," IEEE Trans. Circuits Syst. II, Analog Digit. Signal Process., vol. 46, no. 1, pp. 78-80, 1999.

[3] T. Chen, K. Ma, L. Chen, "Tri-state median filter for image denoising," IEEE Trans Image Process., vol. 8, no. 12 , pp. 1834-1838,1999.

[4] T. Chen, H.R, Wu, "Adaptive impulse detection using center-weighted median filters," IEEE Signal Process. Lett., vol. 8, no. 1, pp.1-3, 2001.

[5] S. Zhang, M. A. Karim, “A new impulse detector for switching median filters," IEEE Signal Process. Lett., vol. 9, no. 11, pp.360-363, 2002.

[6] V. Crnojevic, V. Senk, Z. Trpovski, "Advanced impulse detection based on pixel-wise MAD," IEEE Signal Process. Lett., vol. 11, no. 7, pp.589-592, 2004.

[7] K.S. Srinivasan, D. Ebenezer, "A new fast and efficient decision-based algorithm for removal of highdensity impulse noises,” IEEE Signal Process Lett., vol. 14, no.3, pp.189-192, 2007.

[8] Z. Deng, Z. Yin, Y. Xiong, "High probability impulse noise-removing algorithm based on mathematical morphology,” IEEE Signal Process. Lett. , vol. 14, no.1, pp. 31-34, 2007.

[9] K. Zhan, H.J. Zhang, Y.D. Ma, "New spiking cortical model for invariant texture retrieval and image processing," IEEE Trans. on neural networks, vol. 20, no. 12, pp.1980-1986,2009. 